\title{
Psychological characteristics of adult neurofibromatosis type 1 patients seeking elective surgery
}

Kyunghyun Min ${ }^{1}$, Dae Won Hong ${ }^{1}$, Eun Key Kim ${ }^{1}$, Beom Hee Lee ${ }^{2,3}$

${ }^{1}$ Department of Plastic Surgery, Asan Medical Center, University of Ulsan College of Medicine, Seoul; ${ }^{2}$ Department of Pediatrics and ${ }^{3}$ Medical Genetics Center, Asan Medical Center Children's Hospital, University of Ulsan College of Medicine, Seoul, Korea
We sincerely thank Professor Jung-Bok Lee, Division of Biostatistics, Center for Medical Research and Information, Asan Medical Center, University of Ulsan College of Medicine, for support with the statistical analysis and professional advice.
Background Neurofibromatosis type 1 (NF1) is an autosomal dominant genetic disorder involving the nervous system characterized by the development of neurofibromas throughout the body. Patients with NF1 are also known to have cognitive, behavioral, social, and emotional problems. Using the Symptom Checklist-90-Revision (SCL-90-R) questionnaire, we aimed to assess the psychological characteristics of NF1 patients seeking surgery at a tertiary hospital.

Methods Adult NF1 patients visiting a plastic surgery outpatient clinic between March 2018 and March 2020 were enrolled in this study. The presence and intensity of psychopathological symptoms were assessed using 10 domains, including the General Severity Index (GSI). Standard T-scores were used to compare the results to population-based norms. The impact of demographic factors was also analyzed.

Results In total, 65 patients were included in the study. The mean GSI of all patients was 43.7 , and the mean score of the other nine domains was 45.3. No scores deviated from the population-based normal ranges. Nonetheless, women had significantly higher mean T-scores than men in a few domains, including the GSI, obsessive-compulsive disorder, depression, and anxiety. Most of the other characteristics, such as age, education, marital status, family history, and tumor site did not have significant effects.

Conclusions Adult NF1 patients who visit plastic surgery clinics for elective surgery have psychopathological characteristics that do not differ from the general population according to the SCL-90-R. The results of this study can be considered in consultations with these patients.

Keywords Neurofibromatosis 1 / Psychology / Elective surgical procedures

\section{INTRODUCTION}

Neurofibromatosis type 1 (NF1) is an autosomal dominant genetic disorder involving the nervous system, caused by a heterozygous mutation of the NF1 tumor suppressor gene on chromosome 17. It affects approximately 1 in 2,500 to 3,000 individuals, with an equal

Received: Jun 12, 2020 Revised: Jul 14, 2020 Accepted: Jul 21, 2020 Correspondence: Eun Key Kim Department of Plastic Surgery, Asan Medical Center, University of Ulsan College of Medicine, 88 Olympic-ro 43-gil, Songpa-gu, Seoul 05505, Korea

Tel: +82-2-3010-3600, Fax: +82-2-476-7471, E-mail: nicekek@korea.com

Copyright @ 2020 The Korean Society for Aesthetic Plastic Surgery.

This is an Open Access article distributed under the terms of the Creative Commons Attribution Non-Commercial License (https://creativecommons.org/licenses/by-nc/4.0/) which permits unrestricted non-commercial use, distribution, and reproduction in any medium, provided the original work is properly cited. www.e-aaps.org sex distribution [1]. As implied by the name of the disease, neurofibroma development is one of its major clinical features. Neurofibromas are tumors of peripheral nerve sheath origin. They can appear in any part of the body, with variable clinical and pathological manifestations. Generally, their presentations range from the most common localized forms to complex diffuse or plexiform types [2]. Moreover, a rare malignant form also exists, characterized by tumors known as malignant peripheral nerve sheath tumors. Otherwise, neurofibromas are basically benign tumors, although they can arise virtually anywhere that nervous tissue exists and cause sensory or motor symptoms. In some cases, significant morbidity or even death occurs, depending on the location or tumor burden. Even in cases without considerable morbidity, the disfigurement caused by multiple cutaneous neurofibromas or a few huge plexiform neurofibromas can cause profound distress to the individuals affected. 
As for the cognitive and emotional aspects of NF1, it has been reported that children and adolescents with $\mathrm{NF} 1$ present with significant alterations in language skills, motor function, executive function, attention, behavior, emotion, and social skills. A high prevalence of attention-deficit/hyperactivity disorder, autistic traits, and autistic spectrum disorder have also been reported in association with NF1 $[3,4]$. Compared with unaffected children and adolescents in the general population, pediatric and adolescent patients with NF1 have an increased risk of exhibiting social difficulties, mental health disorders, and behavioral and emotional problems, as well as a diminished quality of life (QOL) [5]. In a crosssectional study by Cohen et al. [6], 55\% of adult NF1 patients likely had clinical depression.

The main objective of this study was to assess, using the Symptom Checklist-90-Revision (SCL-90-R), the psychological characteristics of NF1 patients seeking plastic surgery at a tertiary hospital. Furthermore, the impact of various factors, such as sex, age, education, family history, and tumor location was analyzed.

\section{METHODS}

This cross-sectional study included NF1 patients visiting a plastic surgery outpatient clinic at a tertiary hospital in Korea between March 2018 and March 2020. Eligible patients were $\geq 18$ years old, had already received or intended to undergo surgery for benign neurofibromas, and had completed the SCL-90-R questionnaire. This study was approved by the hospital's institutional review board (2019-1462). The presence and intensity of psychopathological symptoms were assessed using the SCL-90-R, which covers nine primary symptom dimensions: somatization, obsessive-compulsive disorder, interpersonal sensitivity, depression, anxiety, hostility, phobic anxiety, paranoid ideation, and psychoticism. The
SCL-90-R comprises 90 items, which are scored on a scale from 0 to 4 ( 0 , not at all; 1 , a little bit; 2 , moderately; 3 , quite a bit; and 4 , extremely). To assess the clinical relevance of the findings, the SCL90-R scores were converted to standard T-scores (ranging from 30 to 80 ) by referring to the appropriate population-based norm tables provided by the test manual. A T-score of 50 represents the mean $\mathrm{T}$-score of the general population, and $\mathrm{T}$-scores ranging from 40 to 60 represent the normal range (as defined by mean \pm standard deviation). A T-score of 63 corresponds to the 90th percentile and is clinically meaningful; a T-score of 55 is considered relatively high. Table 1 presents definitions of the psychopathological symptoms and disorders measured by the SCL-90-R [7,8].

Statistical analyses were performed using SPSS Statistics for Windows, version 24.0 (IBM Corp., Armonk, NY, USA). The twosample t-test was conducted to assess differences between two independent groups. For three-group comparisons, analysis of variation was performed to evaluate the statistical significance of differences between means. Statistical significance was confirmed by Pvalues $<0.05$. The psychopathological characteristics of the entire patient group were analyzed and compared with the populationbased norms using standardized T-scores with no control group.

\section{RESULTS}

In total, 65 patients completed the SCL-90-R questionnaire. The mean T-score for all patients was $43.7 \pm 11.7$, which was within the normal range, and no subscales deviated from the populationbased norms (Table 2).

Women had a significantly higher mean General Severity Index (GSI) T-score $(45.37 \pm 12.86)$ than men $(41.80 \pm 9.78$; $\mathrm{P}=0.027)$. Moreover, women had significantly higher mean T-scores than men for the dimensions dealing with obsessive-compulsive disor-

Table 1. Description of subscales in the Symptom Checklist-90-Revision (SCL-90-R)

\begin{tabular}{|c|c|}
\hline Scale & What does the scale measure? \\
\hline Somatization & $\begin{array}{l}\text { Discomfort caused by somatic ailments characteristic of neurosis (questions about, e.g., symptoms involving the myocardium, } \\
\text { respiratory system, stomach, myalgia) }\end{array}$ \\
\hline Obsessive-compulsive disorder & $\begin{array}{l}\text { Presence of obsessive-compulsive thoughts and compulsive activities; this scale also comprises more general cognitive } \\
\text { impairments (e.g., mind going blank, recollection of problems) }\end{array}$ \\
\hline Interpersonal sensitivity & $\begin{array}{l}\text { Feeling of interpersonal inadequateness, inferiority, tendency towards self-deprecation, discomfort in social situations } \\
\text { (hypersensitivity, negative expectations about interpersonal communication) }\end{array}$ \\
\hline Depression & $\begin{array}{l}\text { Bad mood, dysphoria, anhedonia, loss of interest, loss of energy and motivation, feeling of helplessness and hopelessness, } \\
\text { suicidal thoughts; this scale also comprises cognitive and somatic symptoms of depression }\end{array}$ \\
\hline Anxiety & $\begin{array}{l}\text { Anxiety, irritability, tension, also somatic symptoms of anxiety, such as palpitation, excitement, questions about acute and } \\
\text { general anxiety }\end{array}$ \\
\hline Hostility & Irritability, annoyance, predisposition to impulsive destruction of objects and frequent uncontrollable outbursts of anger \\
\hline Phobic anxiety & Episodes of acute anxiety states and agoraphobia (fear of travelling, open spaces, crowds, public places) \\
\hline Paranoid ideation & Suspicion, hostility, mistrust towards others, projective thinking \\
\hline Psychoticism & $\begin{array}{l}\text { Auditory hallucinations, transmission and insertion of thoughts, controlling thoughts from the outside and indicators of the } \\
\text { schizoid lifestyle }\end{array}$ \\
\hline
\end{tabular}


$\operatorname{der}(\mathrm{P}=0.023)$, depression $(\mathrm{P}=0.012)$, and anxiety $(\mathrm{P}=0.027)(\mathrm{Ta}-$ ble 3).

Older patients had higher mean T-scores than younger patients ( $49.20 \pm 8.10$ for age $\geq 50$ years vs. $41.25 \pm 12.68$ for patients in their 20s) (Table 4). However, no statistically significant correlations

Table 2. Overall T-scores $(n=65)$

\begin{tabular}{ll}
\hline Domain & T-score \\
\hline GSI total & $43.7 \pm 11.7$ \\
SOM & $45.6 \pm 8.5$ \\
OC & $45.6 \pm 11.2$ \\
IS & $45.7 \pm 10.9$ \\
DEP & $44.1 \pm 11.7$ \\
ANX & $45.9 \pm 9.3$ \\
HOS & $45.7 \pm 10.3$ \\
PHOB & $46.5 \pm 6.1$ \\
PAR & $44.4 \pm 9.1$ \\
PSY & $44.2 \pm 8.6$ \\
\hline
\end{tabular}

Values are presented as mean \pm SD.

GSI, Global Severity Index; SOM, somatization; OC, obsessive-compulsive disorder; IS, interpersonal sensitivity; DEP, depression; ANX, anxiety; HOS, hospitality; PHOB, phobic anxiety; PAR, paranoid ideation; PSY, psychoticism.

Table 4. Comparisons by age

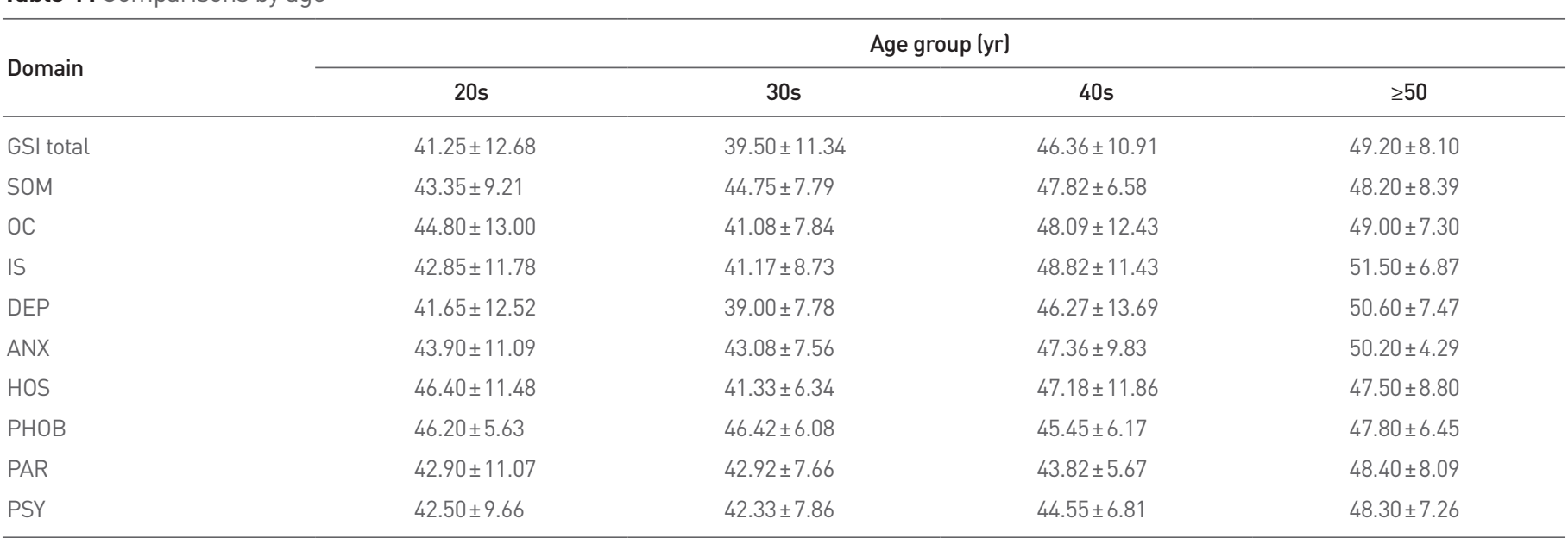

Values are presented as mean \pm SD.

GSI, Global Severity Index; SOM, somatization; OC, obsessive-compulsive disorder; IS, interpersonal sensitivity; DEP, depression; ANX, anxiety; HOS, hospitality; PHOB, phobic anxiety; PAR, paranoid ideation; PSY, psychoticism.

Table 5. Correlation coefficients according to age

\begin{tabular}{|c|c|c|c|c|c|c|c|c|c|c|}
\hline & GSI total & SOM & $\mathrm{OC}$ & IS & DEP & ANX & HOS & РHOB & PAR & PSY \\
\hline P-value & 0.191 & 0.098 & 0.415 & 0.080 & 0.146 & 0.319 & 0.948 & 0.32 & 0.449 & 0.273 \\
\hline
\end{tabular}

GSI, Global Severity Index; SOM, somatization; OC, obsessive-compulsive disorder; IS, interpersonal sensitivity; DEP, depression; ANX, anxiety; HOS, hospitality; PHOB, phobic anxiety; PAR, paranoid ideation; PSY, psychoticism. 
score among patients who had less than a high school diplomalevel education $(47.60 \pm 8.79)$ was higher than those of the other two education-level groups, but these differences were not statistically significant $(\mathrm{P}=0.628)$ (Table 6).

Married patients had higher mean T-scores than unmarried patients for depression $(45.60 \pm 9.28$ vs. $43.45 \pm 12.50$, respectively; $\mathrm{P}=0.929)$ and paranoid ideation ( $45.90 \pm 7.47$ vs. $43.75 \pm 9.61$, respectively; $\mathrm{P}=0.916)$. For hostility, married patients $(44.60 \pm 8.91)$ had lower meant T-scores than unmarried patients $(46.20 \pm 10.81$, $\mathrm{P}=0.361$ ). None of these differences were statistically significant (Table 7).
Among the patients who answered the questionnaire, those with a family history of NF1 scored lower than those without a family history, except for somatization. Hostility was the only category for which family history was associated with a significant difference in mean T-scores (family-history group, 42.90 \pm 7.57 ; no-family-history group, $48.20 \pm 11.68 ; \mathrm{P}=0.042$ ) (Table 8 ).

Lastly, patients with tumors on the face and neck had higher mean T-scores for obsessive-compulsive disorder (face and neck, $48.32 \pm 11.72$; extremity, $44.06 \pm 7.58$; trunk, $42.80 \pm 12.40$ ), interpersonal sensitivity (face and neck, $48.24 \pm 11.41$; extremity, $42.94 \pm$ 8.00, trunk, $44.30 \pm 11.90$ ), depression, anxiety, and hostility. Using

Table 6. Comparisons by educational status

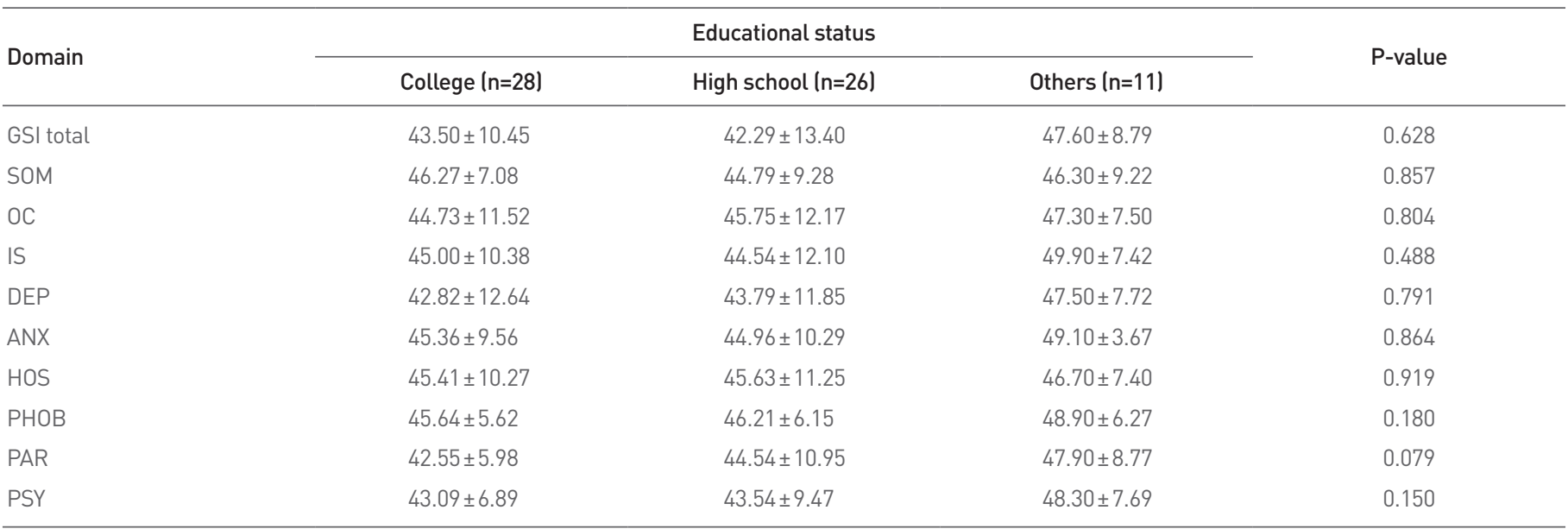

Values are presented as mean \pm SD.

GSI, Global Severity Index; SOM, somatization; OC, obsessive-compulsive disorder; IS, interpersonal sensitivity; DEP, depression; ANX, anxiety; HOS, hospitality; PHOB, phobic anxiety; PAR, paranoid ideation; PSY, psychoticism.

Table 7. Comparisons by marital status

\begin{tabular}{llll}
\hline \multirow{2}{*}{ Domain } & \multicolumn{2}{c}{ Marriage } & \\
\cline { 2 - 3 } & No (n=28) & Pes (n=37) & \\
\hline GSI total & $43.73 \pm 11.63$ & $43.70 \pm 12.00$ & 0.833 \\
SOM & $45.45 \pm 8.39$ & $46.10 \pm 8.81$ & 0.951 \\
OC & $45.93 \pm 11.93$ & $44.90 \pm 8.96$ & 0.540 \\
IS & $45.35 \pm 11.50$ & $46.50 \pm 9.33$ & 0.998 \\
DEP & $43.45 \pm 12.50$ & $45.60 \pm 9.28$ & 0.929 \\
ANX & $45.68 \pm 9.93$ & $46.30 \pm 7.64$ & 0.694 \\
HOS & $46.20 \pm 10.81$ & $44.60 \pm 8.91$ & 0.361 \\
PHOB & $46.38 \pm 6.02$ & $46.70 \pm 6.22$ & 0.978 \\
PAR & $43.75 \pm 9.61$ & $45.90 \pm 7.47$ & 0.916 \\
PSY & $43.95 \pm 8.94$ & $44.90 \pm 7.56$ & 0.834 \\
\hline
\end{tabular}

Values are presented as mean \pm SD.

GSI, Global Severity Index; SOM, somatization; OC, obsessive-compulsive disorder; IS, interpersonal sensitivity; DEP, depression; ANX, anxiety; HOS, hospitality; PHOB, phobic anxiety; PAR, paranoid ideation; PSY, psychoticism.
Table 8. Comparisons by family history of neurofibromatosis 1

\begin{tabular}{lccc}
\hline \multirow{2}{*}{ Domain } & \multicolumn{2}{c}{ Family history } & \\
\cline { 2 - 3 } & No $(\mathrm{n}=34)$ & Pes $(\mathrm{n}=31)$ & \\
\hline GSI total & $45.37 \pm 12.24$ & $41.80 \pm 10.8$ & 0.206 \\
SOM & $44.97 \pm 8.746$ & $46.40 \pm 8.18$ & 0.595 \\
OC & $47.63 \pm 12.93$ & $43.30 \pm 8.12$ & 0.107 \\
IS & $47.53 \pm 11.85$ & $43.50 \pm 9.33$ & 0.207 \\
DEP & $45.50 \pm 13.60$ & $42.40 \pm 8.77$ & 0.133 \\
ANX & $47.03 \pm 10.30$ & $44.50 \pm 7.87$ & 0.232 \\
HOS & $48.20 \pm 11.68$ & $42.90 \pm 7.57$ & $0.042^{\mathrm{a}}$ \\
PHOB & $47.23 \pm 6.20$ & $45.60 \pm 5.81$ & 0.748 \\
PAR & $45.00 \pm 10.43$ & $43.60 \pm 7.21$ & 0.628 \\
PSY & $45.73 \pm 9.30$ & $42.50 \pm 7.29$ & 0.626 \\
\hline
\end{tabular}

Values are presented as mean $\pm \mathrm{SD}$.

GSI, Global Severity Index; SOM, somatization; OC, obsessive-compulsive disorder; IS, interpersonal sensitivity; DEP, depression; ANX, anxiety; HOS, hospitality; PHOB, phobic anxiety; PAR, paranoid ideation; PSY, psychoticism. a) Statistically significant, $\mathrm{P}<0.05$. 
Table 9. Comparisons by the main bodily region of the disease

\begin{tabular}{llcc}
\hline Domain & $\begin{array}{c}\text { Face and neck } \\
(\mathbf{n}=38)\end{array}$ & $\begin{array}{c}\text { Extremity } \\
(\mathbf{n}=32)\end{array}$ & $\begin{array}{c}\text { Trunk } \\
(\mathbf{n}=24)\end{array}$ \\
\hline GSI total & $46.00 \pm 12.06$ & $42.75 \pm 9.28$ & $40.90 \pm 12.8$ \\
SOM & $46.16 \pm 9.63$ & $45.25 \pm 6.06$ & $45.20 \pm 8.75$ \\
OC & $48.32 \pm 11.72$ & $44.06 \pm 7.58$ & $42.80 \pm 12.40$ \\
IS & $48.24 \pm 11.41$ & $42.94 \pm 8.00$ & $44.30 \pm 11.90$ \\
DEP & $47.32 \pm 11.66$ & $42.50 \pm 8.83$ & $40.30 \pm 13.00$ \\
ANX & $47.20 \pm 10.42$ & $46.75 \pm 6.35$ & $42.70 \pm 9.34$ \\
HOS & $47.36 \pm 12.77$ & $43.25 \pm 6.02$ & $45.70 \pm 8.78$ \\
PHOB & $47.04 \pm 6.02$ & $47.31 \pm 5.99$ & $44.60 \pm 5.87$ \\
PAR & $45.68 \pm 10.34$ & $43.25 \pm 6.14$ & $43.30 \pm 9.25$ \\
PSY & $45.04 \pm 9.52$ & $43.94 \pm 6.66$ & $43.10 \pm 8.62$ \\
\hline
\end{tabular}

Values are presented as mean \pm SD.

GSI, Global Severity Index; SOM, somatization; OC, obsessive-compulsive disorder; IS, interpersonal sensitivity; DEP, depression; ANX, anxiety; HOS, hospitality; PHOB, phobic anxiety; PAR, paranoid ideation; PSY, psychoticism.

the two-sample t-test, it was found that patients with face and neck tumors had significantly higher mean T-scores for depression $(\mathrm{P}=0.03)$ and hostility $(\mathrm{P}=0.047)$ than patients with tumors affecting other body sites. The differences associated with obsessivecompulsive disorder $(\mathrm{P}=0.051)$ and anxiety $(\mathrm{P}=0.074)$ score were marginally significant, and the differences associated with the other analyzed symptoms and disorders were not statistically significant (Table 9).

\section{DISCUSSION}

The SCL-90-R is a self-report mental health questionnaire mainly used to evaluate a patient's psychological state. It is short, easy to use, has been used in many studies, and the Korean-translated version is well established [7-9]. The reliability and validity of the SCL$90-\mathrm{R}$ has been proven in various patient groups. This test has been used to interpret the causes and consequences of various diseases, since the questions are simple and can be applied to a wide range of patients [10-15]. When applying and interpreting the SCL-90-R, it is common to compare the scores of patients and controls, but the tool can also be used to compare patients' scores with population-based norms using standard T-scores, an approach that has been utilized in other regions with their own population-based norms [16]. We analyzed the results using standardized T-scores based on Korean non-patient population data collected in 2014 from 1,000 normative people (501 males and 499 females) based on the population distribution by region and internet usage by age. Standard T-scores were also verified by age distribution and education level. A T-score of 63 corresponds to the 90th percentile and is clinically meaningful, and a T-score of 55 is considered relatively high. This enables within- and across-profile interpretation with- out a control group [17].

In general, it has been reported that NF1 patients commonly show neurodevelopmental abnormalities in various domains and to various degrees. For example, impairments in general cognitive/ executive function, learning and social skills, anxiety and depression are associated with NF1 [4-6]. This is more than a social myth that comes from appearance, because these impairments are caused by pathogenic variants in genes encoding the Ras-mitogenactivated protein kinase signaling pathway [18]. These neurological and emotional features have significant negative impacts on QOL $[19,20]$. In the NF1 patient group of our study, however, there was no significant cognitive or emotional deviation relative to the general population. Except for sex, the demographic variables did not affect any of the SCL-90-R domains, including the GSI. Women are generally considered to be at a higher risk of mood disorders, even among non-neurofibromatosis patients. In fact, women have been reported to score higher in every subscale of the SCL90-R except psychoticism (although the difference was not statistically significant for the psychoticism domain, as it was for somatization) [21], and Ablon [22] also described differences in responses to NF1 by sex, claiming that attitudes and social lifestyles, as well as the effects of changes in appearance on marriage or occupational outcomes, differ by sex.

Even considering these aspects, the results of our study significantly differed from those of previous publications. There are some important points to consider when interpreting the results. In terms of academic background, the majority of the study patients had at least an undergraduate-level education, and among the high school graduates, there were many current college students. Additionally, half of the unmarried patients were still young, in their 20s and 30s. This could be explained by selection bias, which is an intrinsic limitation of our study. In other words, among all individuals with NF1, our patients were those who visited a tertiary hospital for the purpose of surgery and who had the intellectual capacity and desire to fill out the questionnaire. This limits the generalizability of our results to all NF1 patients. Nonetheless, these patients are, broadly speaking, representative of the NF1 patients encountered at plastic surgery clinics.

A few studies have analyzed the relationship between QOL and the tumors themselves among NF1 patients. One study found that tumor burden was only correlated with pain, and not general QOL [23]. However, Granstrom et al. [24] claimed that adult NF1 patients had negative body image and less self-confidence, and that body image was an important link between disease visibility and psychological well-being. A similar perspective was also reflected by Taylor and Lewis [25], who asserted that surgery for NF1 is not cosmetic; rather, it is restoration of a normal appearance from disfigurement. However, considering the disease entity and the patient characteristics revealed by our results, elective surgery for NF1 is similar to aesthetic surgery in some respects. Therefore, when con- 
sulting with patients and deciding on surgery for NF1 (especially the cutaneous type), it is necessary to consider these issues and to clearly discuss the motivations for surgery, as well as its limitations.

The most important limitations of this study are that was based on a self-report questionnaire, making many of the findings subjective, and that there was inherent selection bias caused by enrolling only NF1 patients who visited a plastic surgery outpatient clinic at a tertiary hospital and who were able and willing to complete a questionnaire. The study would have been more comprehensive and objective if we had compared the patients to a non-patient group or another benign tumor patient group. Nevertheless, to our knowledge, this was the first study to describe the psychopathological features of selected NF1 patients seeking elective surgery.

In conclusion, according to the SCL-90-R findings, adult NF1 patients who voluntarily seek elective surgery have psychopathological characteristics that do not differ from the general population. Women had significantly higher mean T-scores than men in some domains, including the GSI, obsessive-compulsive disorder, depression, and anxiety, but all T-scores were within normal ranges. Features such as age, education, marital status, family history, and tumor site mostly did not have significant effects. These results should be considered when considering surgery for such patients.

\section{NOTES}

\section{Conflict of interest}

No potential conflict of interest relevant to this article was reported.

\section{Ethical approval}

The study was approved by the Institutional Review Board of Asan Medical Center (IRB No. 2019-1462) and performed in accordance with the principles of the Declaration of Helsinki.

\section{Patient consent}

The patients provided written informed consent for the publication.

\section{ORCID}

Kyunghyun Min https://orcid.org/0000-0002-7807-0143

Dae Won Hong

Eun Key Kim https://orcid.org/0000-0003-4218-8833

Beom Hee Lee https://orcid.org/0000-0002-3986-6886 https://orcid.org/0000-0001-9709-2631

\section{REFERENCES}

1. Lammert M, Friedman JM, Kluwe L, et al. Prevalence of neurofibromatosis 1 in German children at elementary school enrollment. Arch Dermatol 2005;141:71-4.

2. Ortonne N, Wolkenstein P, Blakeley JO, et al. Cutaneous neurofibromas: current clinical and pathologic issues. Neurology 2018;91:S5-13.

3. Torres Nupan MM, Velez Van Meerbeke A, Lopez Cabra CA, et al.
Cognitive and behavioral disorders in children with neurofibromatosis type 1. Front Pediatr 2017;5:227.

4. Vogel AC, Gutmann DH, Morris SM. Neurodevelopmental disorders in children with neurofibromatosis type 1. Dev Med Child Neurol 2017;59:1112-6.

5. Domon-Archambault V, Gagnon L, Benoit A, et al. Psychosocial features of neurofibromatosis type 1 in children and adolescents. J Child Neurol 2018;33:225-32.

6. Cohen JS, Levy HP, Sloan J, et al. Depression among adults with neurofibromatosis type 1: prevalence and impact on quality of life. Clin Genet 2015;88:425-30.

7. Kim KI, Won HT, Lee JH, et al. Standardization study of symptom check list-90 in Korea I: characteristics of normal responses. J Korean Neuropsychiatr Assoc 1978;17:449-58.

8. Kim JH, Kim GI. The standardization study of Symptom Checklist90-revision in Korea III. Ment Health Res 1984;2:278-311.

9. Kim JH, Kim KI, Lee HR. The code patterns of Korean version of the Symptom Checklist-90-revision. Ment Health Res 1985;3:173-217.

10. Baranyi A, Krauseneck T, Rothenhäusler HB. Overall mental distress and health-related quality of life after solid-organ transplantation: results from a retrospective follow-up study. Health Qual Life Outcomes 2013;11:15.

11. Kostyla M, Tabala K, Kocur J. Illness acceptance degree versus intensity of psychopathological symptoms in patients with psoriasis. Postepy Dermatol Alergol 2013;30:134-9.

12. Kim IH, Kim CY, Kim KH, et al. Analysis of female lichen planus patients with SCL-90-R. J Oral Med Pain 2011;36:235-43.

13. Jin SM, Ban JH, Lee SH, et al. Psychologic charateristics of patients with vocal nodules: an investigation using the Symptom Checklist-90-revision. Korean J Otorhinolaryngol Head Neck Surg 2000;43:86-9.

14. Ko MY, Kim CY, Jeon HM, et al. Psychological analysis of recurrent aphthous ulcer patients with SCL-90-R. J Oral Med Pain 2012;37:1925.

15. Yun YH, Lee SH, Choi JH. Neurocognitive function in patients with Hwa-byung and with major depressive disorder. Korean J Biol Psychiatry 2005;12:181-8.

16. Sereda Y, Dembitskyi S. Validity assessment of the Symptom Checklist SCL-90-R and shortened versions for the general population in Ukraine. BMC Psychiatry 2016;16:300.

17. Won HT, Kim JH, Oh KJ, et al. Korean manual of Symptom Checklist-90 Revision (SCL-90-R). Seoul: Huno; 2015.

18. McNeill AM, Hudock RL, Foy AMH, et al. Emotional functioning among children with neurofibromatosis type 1 or Noonan syndrome. Am J Med Genet A 2019;179:2433-46.

19. Lai JS, Jensen SE, Charrow J, et al. Patient reported outcomes measurement information system and quality of life in neurological disorders measurement system to evaluate quality of life for children and adolescents with neurofibromatosis type 1 associated plexiform neurofibroma. J Pediatr 2019;206:190-6.

20. Ferner RE, Thomas M, Mercer G, et al. Evaluation of quality of life in 
adults with neurofibromatosis 1 (NF1) using the impact of NF1 on quality of life (INF1-QOL) questionnaire. Health Qual Life Outcomes 2017;15:34.

21. Grande TL, Newmeyer MD, Adair ES. Symptom differences by gender for outpatient clients as measured by the SCL-90-R. Michigan J Couns Res 2013;40:4-13.

22. Ablon J. Gender response to neurofibromatosis 1. Soc Sci Med 1996; 42:99-109.

23. Merker VL, Bredella MA, Cai W, et al. Relationship between whole- body tumor burden, clinical phenotype, and quality of life in patients with neurofibromatosis. Am J Med Genet A 2014;164A:1431-7.

24. Granstrom S, Langenbruch A, Augustin M, et al. Psychological burden in adult neurofibromatosis type 1 patients: impact of disease visibility on body image. Dermatology 2012;224:160-7.

25. Taylor LA, Lewis VL Jr. Neurofibromatosis type 1: review of cutaneous and subcutaneous tumor treatment on quality of life. Plast Reconstr Surg Glob Open 2019;7:e1982. 